\title{
Intensity Noise and Linewidth of Laser Diodes With Integrated Semiconductor Optical Amplifier
}

\author{
Geert Morthier, Senior Member, IEEE, and Bart Moeyersoon, Student Member, IEEE
}

\begin{abstract}
It is shown that the relative intensity noise and the linewidth of a laser diode can increase significantly if an optical amplifier is integrated with the laser diode. This increase is due to the amplified spontaneous emission from the optical amplifier.
\end{abstract}

Index Terms-Noise, optical communication, semiconductor lasers.

\section{INTRODUCTION}

C ORE optical networks require transmitter modules capable of delivering high optical power levels. However, many of the more advanced laser diodes used in such transmitter modules are limited in optical power. In order to boost the power levels, a semiconductor optical amplifier (SOA) is sometimes integrated with the laser diodes. This is, e.g., the case for several types of tunable or widely tunable laser diodes [1], [2] as well as for many types of multiwavelength lasers [3]. Widely tunable laser diodes often consist of passive (lossy) grating sections on either side of the active section, which limits the maximum output power and necessitates the use of an integrated semiconductor optical amplifier. Multiwavelength lasers often consist of laser arrays and a combiner in which the light of the individual lasers is combined. The combiner introduces significant losses, which are preferably compensated by an integrated amplifier.

We will show, however, that by integrating an optical amplifier with a laser diode, the noise characteristics of the laser diode can be seriously degraded. Due to the amplified spontaneous emission noise from the amplifier, both the relative intensity noise (RIN) and the laser linewidth can increase with one or more orders of magnitude. This degradation can obviously also occur when a laser diode is connected to a separate SOA or EDFA without isolator in-between. A related configuration, a laser diode directly connected to or integrated with a SOA-based wavelength converter, will suffer from a similar noise degradation.

An expression for the linewidth of the cascade of a laser diode and an optical amplifier has been derived earlier by Berglind and Nilsson [4] using a circuit-theoretical approach. Here, we will describe a more general and physical derivation based on an alternative rate equation description [5] that was published earlier. It leads to expressions for both the linewidth and the RIN.

Manuscript received June 19, 2002; revised August 19, 2002. This work was supported in part by the Belgian Federal Office for Scientific, Technical, and Cultural Affairs via the research network IAP V-18, the PHOTON Network.

The authors are with the Department of Information Technology, Ghent University_IMEC, B-9000 Ghent, Belgium (e-mail: morthier@intec.rug.ac.be).

Digital Object Identifier 10.1109/LPT.2002.804672

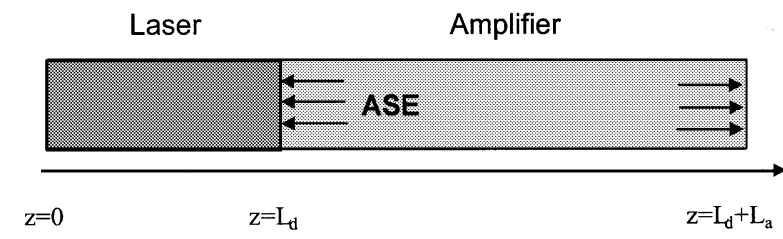

Fig. 1. Schematic structure of a laser diode integrated with a semiconductor optical amplifier.

The alternative rate equation description is briefly introduced in the next paragraph. This is followed by the derivation of linewidth and RIN and a discussion of the obtained results.

\section{RATE EQUATIONS FOR A LASER CONNECTED TO AN OPTICAL AMPLIFIER}

The schematic structure of the laser diode integrated with the SOA is shown in Fig. 1. The laser diode is a general multisection DFB or DBR laser diode and we first derive the rate equations for the laser diode. The influence of the SOA on the laser diode consists of (backward propagating) amplified spontaneous emission noise that is injected in the laser diode at $z=L_{d}$. This spontaneous emission noise is characterized by a Langevin function $F_{i}(t)$, which has the following second-order moment:

$$
\begin{aligned}
& \left\langle F_{i}(L, t) F_{i}^{*}\left(L, t^{\prime}\right)\right\rangle \\
& \quad=\hbar \omega(\Gamma g)_{a} n_{\mathrm{sp}, a} \frac{\exp \left[(\Gamma g-\alpha)_{a} L_{a}\right]-1}{(\Gamma g-\alpha)_{a}} \delta\left(t-t^{\prime}\right)
\end{aligned}
$$

with $L_{a}$ the amplifier length and $(\Gamma g)_{a}, n_{\mathrm{sp}, a}, \alpha_{a}$ the modal gain, inversion factor, and internal loss of the amplifier, respectively. It is assumed that the fields are normalized such that their squared amplitude represents the power.

To derive the alternative rate equations for the laser diode, we follow the procedure outlined in [5]. The complex amplitudes $R^{+}(z, t)$ and $R^{-}(z, t)$ of the forward- and backward-propagating fields and the complex Bragg deviation $\Delta \beta(z, t)$ are expanded as

$$
\begin{aligned}
& R^{ \pm}(z, t)=R_{0}^{ \pm}(z)+\delta R^{ \pm}(z, t) \\
& \Delta \beta(z, t)=\Delta \beta_{0}+\delta(\Delta \beta(z, t))
\end{aligned}
$$

and the expansions are introduced in the time-dependent coupled wave equations (cf. [5]). Combination of these time-dependent coupled wave equations with the static coupled wave equations and integration from $z=0$ (left-hand-side laser facet) 
to $z=L_{d}$ (right-hand-side laser facet) yields the following equation:

$$
\begin{aligned}
2 \int_{0}^{L_{d}} & \delta(\Delta \beta) R_{0}^{+} R_{0}^{-} d z \\
= & \frac{j}{v_{g}} \frac{\partial}{\partial t}\left(\int_{0}^{L_{d}}\left(R_{0}^{+} \delta R^{-}+R_{0}^{-} \delta R^{+}\right) d z\right) \\
& \quad-j \int_{0}^{L_{d}}\left(R_{0}^{-} F^{+}+R_{0}^{+} F^{-}\right) d z-t_{2} R_{0}^{+}\left(L_{d}\right) F_{i}(t)
\end{aligned}
$$

with $t_{2}$ the field transmission of the right-hand-side facet. The second term on the right-hand side represents the spontaneous emission generated in the laser cavity. It is now assumed that the spatial profiles of $\delta R^{+}$and $\delta R^{-}$are the same as the spatial profiles of the static fields such that the following expansions [defining $f(t)$ ] can be written

and

$$
\delta R^{ \pm}(z, t)=R_{0}^{ \pm}(z)\{\sqrt{1+f(t)}-1\}
$$

$$
\delta S(z, t)=S_{0}(z) f(t)
$$

where $S(z)$ is the photon density in the cavity. After introducing the longitudinal confinement factor $\Gamma_{z}(z)$, one then arrives at the general (complex) rate equation for $f$. If, for the sake of simplicity, the nonlinear gain is neglected and the carrier density expanded as $N(z, t)=N_{0}(t)+N_{1}(t)\left[\left(S_{0}(z)-S_{\text {av }}\right) / S_{\text {av }}\right]$, with $S_{\text {av }}$ the spatial average of $S_{0}$, one obtains

$$
\begin{aligned}
& j \frac{\partial \sqrt{1+f}}{\partial t}+v_{g} F_{c}-j v_{g} t \frac{R_{0}^{+}\left(L_{d}\right) F_{i}(t)}{2 \int_{0}^{L_{d}} d z R_{0}^{+} R_{0}^{-}} \\
& \quad=\sqrt{1+f}\left\{\Delta \omega+j \frac{1+j \alpha_{H}}{2} G_{1}^{\prime}\left[\delta N_{0}+\delta N_{1}(\chi-1)\right]\right\}
\end{aligned}
$$

with $G_{l}^{\prime}$ the modal differential gain (per time unit) of the laser waveguide and $\alpha_{H}$ the linewidth enhancement factor. $\chi$ is a complex factor that describes the influence of spatial hole burning and is a function of the field distributions in the laser cavity only [5]. For future use, we denote the third term on the left-hand side as $-j v_{g} F_{\text {inj. }}$. The second-order moment of $v_{g} F_{\text {inj }}$ is given by

$$
\begin{aligned}
& \left\langle v_{g} F_{\mathrm{inj}}(t) F_{\mathrm{inj}}^{*}\left(t^{\prime}\right) v_{g}\right\rangle \\
& =(\Gamma g)_{a} n_{\mathrm{sp}, \mathrm{a}} v_{g} \frac{A-1}{\ln (A)} \frac{K_{z}}{S_{\mathrm{av}} V_{\mathrm{act}}} \\
& \quad \times\left\{\frac{\left|R_{0}^{+}\left(L_{d}\right)\right|^{2}\left|t_{2}\right|^{2}}{\frac{1}{L_{d}} \int_{0}^{L_{d}}\left[\left|R_{0}^{+}\right|^{2}+\left|R_{0}^{-}\right|^{2}\right] d z}\right\} \delta\left(t-t^{\prime}\right)
\end{aligned}
$$

with $A$ the total (net) amplification of the SOA. The last factor of the right-hand side (i.e., the factor between $\{\cdot\}$ ) is again a factor that depends on the laser structure only; it is the extraction efficiency from the right-hand-side facet (denoted further as $\alpha_{r} L_{d}$ ) and is, for AR-coated lasers, generally very close to unity. The second-order moment of $v_{g} F_{c}$ is given by

$$
\left\langle v_{g} F_{c}(t) F_{c}^{*}\left(t^{\prime}\right) v_{g}\right\rangle=(\Gamma g)_{l} n_{\mathrm{sp}, 1} v_{g} \frac{K_{z}}{S_{\mathrm{av}} V_{\mathrm{act}}} \delta\left(t-t^{\prime}\right)
$$

with $V_{\text {act }}$ the active layer volume and $n_{s p, l}$ the inversion factor of the laser. $K_{z}$ is the longitudinal Petermann factor [5]. The rate equation (5) has to be combined with the carrier density rate equations for the derivation of laser characteristics. We will not provide the derivation of the rate equations for $N_{0}$ and $N_{1}$ here and refer to [5].

\section{DERIVATION OF LINEWIDTH AND RELATIVE-INTENSITY NOISE}

To simplify the derivation further, we neglect spatial hole burning from now on and assume that the carrier density in the laser cavity is uniform. We also ignore the shot noise term in the carrier density rate equation. This implies that our derivation of the RIN will only be valid at low or moderate power levels. The rate equation for $N_{0}$ is

$$
\frac{d N_{0}}{d t}=\frac{J}{q d}-\frac{N_{0}}{\tau\left(N_{0}\right)}-G_{1}\left(N_{0}\right) S_{\mathrm{av}}+S_{\mathrm{av}} \operatorname{Im}\left(2 v_{g} F_{c}\right) .
$$

The RIN can be derived from the small signal, frequency-dependent solutions, i.e., after Fourier transform of (8) and of the real and imaginary part of (5). We find [with $(F \Omega)$ the Fourier transform of $f(t)$ ]

$$
\begin{aligned}
F(\Omega) & \left\{j \Omega\left(j \Omega+\frac{}{\tau_{d}}+G_{l}^{\prime} S_{\mathrm{av}}\right)+G_{l}^{\prime} G_{l}\left(N_{0}\right) S_{\mathrm{av}}\right\} \\
= & \left(j \Omega+\frac{1}{\tau_{d}}+G_{l}^{\prime} S_{\mathrm{av}}\right) \operatorname{Re}\left(2 v_{g} F_{\mathrm{inj}}\right) \\
& -\left(j \Omega+\frac{1}{\tau_{d}}\right) \operatorname{Im}\left(2 v_{g} F_{c}\right)
\end{aligned}
$$

which gives for the RIN $\left(\mathrm{RIN}=\left\langle|F|^{2}\right\rangle\right)$

$$
\begin{aligned}
|F(\Omega)|^{2} & \frac{4\left|j \Omega+\frac{1}{\tau_{d}}\right|^{2} G_{l}\left(N_{0}\right) n_{\mathrm{sp}, 1}}{\left|\left\{j \Omega\left(j \Omega+\frac{1}{\tau_{d}}+G_{l}^{\prime} S_{\mathrm{av}}\right)+G_{l}^{\prime} G_{l}\left(N_{0}\right) S_{\mathrm{av}}\right\}\right|^{2}} \frac{K_{z}}{S_{\mathrm{av}} V_{\mathrm{act}}} \\
& \times\left[1+\frac{A-1}{\ln (A)} \frac{L_{a}}{L_{d}} \frac{n_{\mathrm{sp}, \mathrm{a}} \Gamma_{a} g_{a}}{n_{\mathrm{sp}, 1} \Gamma_{l} g_{l}} \alpha_{r} L_{d} \frac{\left|j \Omega+\frac{1}{\tau_{d}}+G_{l}^{\prime} S_{\mathrm{av}}\right|^{2}}{\left|j \Omega+\frac{1}{\tau_{d}}\right|^{2}}\right] .
\end{aligned}
$$

It must emphasized that (10) gives the RIN as would be measured just outside the laser, e.g., at the left-hand-side facet of the chip $(z=0)$. At the right-hand-side facet of the chip $(z=$ $L_{d}+L_{a}$ ), i.e., after the amplifier, the RIN that is measured is modified further by the beat noise between laser signal and the forward propagating ASE from the amplifier. This extra RIN has been described before and is usually negligible [6]. If furthermore the SOA is saturated, then the intensity noise from the right-hand-side laser facet will be suppressed in the SOA.

From a similar derivation, but assuming only low frequencies $\Omega$, one finds for the linewidth

$$
\begin{aligned}
\Delta \nu= & \frac{G_{l} n_{\mathrm{sp}, 1} K_{z}}{4 \pi S_{\mathrm{av}} V_{\mathrm{act}}} \\
& \times\left(1+\alpha_{H}^{2}\right)\left[1+\frac{A-1}{\ln (A)} \frac{L_{a}}{L_{d}} \frac{n_{\mathrm{sp}, \mathrm{a}} \Gamma_{a} g_{a}}{n_{\mathrm{sp}, 1} \Gamma_{l} g_{l}} \alpha_{r} L_{d}\right] .
\end{aligned}
$$


It can be noticed that the formula for the linewidth contains the material linewidth enhancement factor $\alpha_{H}$ instead of the effective linewidth enhancement factor. As has been explained in [5], this is due to the neglect of the spatial hole burning. Inclusion of spatial hole burning (i.e., an equation for $\delta N_{l}$ ) in the derivation would readily yield the effective linewidth enhancement factor in (11).

One sees that both the RIN spectrum and the linewidth of the solitary laser are increased by the presence of the SOA with the factor between $[\cdot]$. The linewidth and RIN increase due to the ASE noise from the optical amplifier can easily be estimated. We consider a $\lambda / 4$-shifted DFB laser, for which $\alpha_{r} L_{d}=1$ (i.e., $\kappa L \approx 1.25$ ). We also assume that laser diode and semiconductor optical amplifier have equal length, equal gain and equal inversion factors. In that case, both the RIN and the linewidth are increased by a factor $1+(A-1) / \ln (A)$. This factor is around $5(7 \mathrm{~dB})$ for an amplification of $10(10 \mathrm{~dB})$, but increases to 22.5 $(13 \mathrm{~dB})$ for an amplification of $20 \mathrm{~dB}$.

\section{NUMERICAL EXAMPLE}

We have verified this theory using the laser simulation software Componentmaker from VPI. To this end, we calculated the RIN of a 400- $\mu$ m-long $\lambda / 4$-shifted, AR-coated DFB laser with and without additional SOA at the right-hand-side facet. The laser has a $\kappa L$ of 2 and is biased such that the output power from the laser (e.g., from the left-hand-side facet) was $0.6 \mathrm{~mW}$. The SOA is $350 \mu \mathrm{m}$ long and gives an amplification of $16 \mathrm{~dB}$. The inversion factor of both laser and SOA is 2 . The RIN as would be measured on the left-hand-side facet is shown in Fig. 2 for the solitary laser as well as for the laser with integrated SOA.

From (10), one finds that the RIN of the laser increases by $11.5 \mathrm{~dB}$ due to the ASE from the SOA. This is very similar to what is obtained from the numerical simulations, especially at low frequencies. At very high frequencies, the simulations predict a RIN increase with $15 \mathrm{~dB}$, i.e., $3 \mathrm{~dB}$ more than expression (10). This discrepancy could be due to, e.g., our simplification of the carrier density dynamics and the neglect of the carrier density shot noise. We have also neglected the dc contribution from the amplifier's ASE injected into the laser, which may explain the difference in damping of the relaxation oscillation.

The expression for the linewidth increase is equivalent to [4, Eq. (13)]. The quantities $G, \eta_{r}$, and $\eta_{\text {ia }}$ in (13) are equivalent to, respectively, $A, \alpha_{r} /\left(\Gamma_{l} g_{l}\right)$, and $\left(\Gamma_{a} g_{a}-\alpha_{a}\right) /\left(\Gamma_{a} g_{a}\right)$ in our model and substitution immediately shows the equivalence of both linewidth formulae.

\section{CONCLUSION}

We have derived expressions for the linewidth and RIN of a laser diode integrated with an optical amplifier. The expressions have been derived from previously derived alternative and more generally valid rate equations and are therefore valid under very general conditions. From our derivation, it follows that both the linewidth and the RIN can increase significantly due to the ASE-noise from the amplifier injected into the laser diode. This

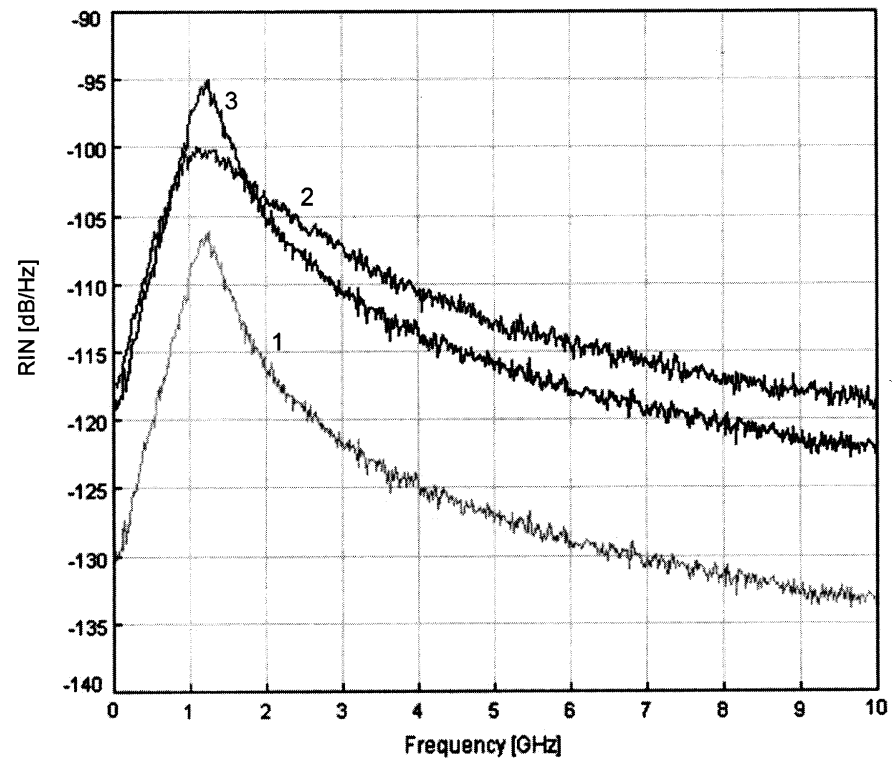

Fig. 2. RIN (as would be measured at the left-hand-side facet) of a $\lambda / 4$-shifted DFB laser with and without integrated SOA on the right-hand-side facet for a laser facet output power of $0.6 \mathrm{~mW}$. (1) without SOA and calculated using Componentmaker from VPI, (2) with SOA and calculated using Componentmaker from VPI, and (3) with SOA and obtained from (1) using formula (10).

is especially the case for large amplification factors. The increase of RIN and phase noise is found both for low and high frequencies.

The large influence of the ASE-noise from the amplifier on the RIN of the laser diode only exists at low to moderate power levels. At high-power levels, the laser RIN is completely dominated by the shot noise in the carrier density.

\section{ACKNOWLEDGMENT}

The authors thank M. Mazed from QDI for supporting this work.

\section{REFERENCES}

[1] B. Mason, J. Barton, G. A. Fish, L. A. Coldren, and S. P. Denbaars, "Design of sampled grating DBR lasers with integrated semiconductor optical amplifiers," IEEE Photon. Technol. Lett., vol. 12, pp. 762-764, July 2000.

[2] J. Gripp, P. Bernasconi, C. Chan, K. L. Sherman, and M. Zirngibl, "Demonstration of a 1Tb/s optical packet switch fabric $(80 * 12.5 \mathrm{~Gb} / \mathrm{s})$, scalable to $128 \mathrm{~Tb} / \mathrm{s}(6400 * 20 \mathrm{~Gb} / \mathrm{s})$," in Proc. ECOC '2000, Munich, Germany, Sept. 2000, Postdeadline Paper PD2.7.

[3] K. Kundo, K. Yashiki, T. Sasaki, Y. Yokoyama, K. Hamamoto, T. Morimoto, and M. Yamaguchi, "1.55- $\mu$ m wavelength-selectable microarray DFB-LD's with monolithically integrated MMI combiner, SOA and EA-modulator," IEEE Photon. Technol. Lett., vol. 12, pp. 242-244, Mar. 2000.

[4] E. Berglind and O. Nilsson, "Laser linewidth broadening caused by a laser amplifier," IEEE Photon. Technol. Lett., vol. 3, pp. 442-444, May 1991.

[5] G. Morthier, "An accurate rate-equation description for DFB lasers and some interesting solutions," IEEE J. Quantum. Electron., vol. 33, pp. 231-237, Feb. 1997.

[6] N. A. Olsson, "Lightwave systems with optical amplifiers," J. Lightwave Technol., vol. 7, pp. 1071-1082, July 1989. 